\title{
Relativistic quantum dynamics of scalar bosons under a full vector Coulomb interaction
}

\author{
Luis B. Castro ${ }^{1, \mathrm{a}}$, Luiz P. de Oliveira ${ }^{2, \mathrm{~b}}$, Marcelo G. Garcia ${ }^{3,4, \mathrm{c}}$, Antonio S. de Castro ${ }^{5, \mathrm{~d}}$ \\ ${ }^{1}$ Departamento de Física, Universidade Federal do Maranhão (UFMA), Campus Universitário do Bacanga, 65080-805 São Luís, MA, Brazil \\ 2 Instituto de Física, Universidade de São Paulo (USP), 05508-900 São Paulo, SP, Brazil \\ ${ }^{3}$ Departamento de Física, Instituto Tecnológico de Aeronáutica (ITA), 12228-900 São José dos Campos, SP, Brazil \\ ${ }^{4}$ Departamento de Matemática Aplicada, Universidade Estadual de Campinas (UNICAMP), IMECC, 13081-970 Campinas, SP, Brazil \\ ${ }^{5}$ Departamento de Física e Química, Universidade Estadual Paulista (UNESP), Campus de Guaratinguetá, 12516-410 Guaratinguetá, SP, Brazil
}

Received: 10 February 2016 / Accepted: 3 May 2017 / Published online: 16 May 2017

(C) The Author(s) 2017. This article is an open access publication

\begin{abstract}
The relativistic quantum dynamics of scalar bosons in the background of a full vector coupling (minimal plus nonminimal vector couplings) is explored in the context of the Duffin-Kemmer-Petiau formalism. The Coulomb phase shift is determined for a general mixing of couplings and it is shown that the space component of the nonminimal coupling is a sine qua non condition for the exact closed-form scattering amplitude. It follows that the Rutherford cross section vanishes in the absence of the time component of the minimal coupling. Bound-state solutions obtained from the poles of the partial scattering amplitude show that the time component of the minimal coupling plays an essential role. The bound-state solutions depend on the nonminimal coupling and the spectrum consists of particles or antiparticles depending on the sign of the time component of the minimal coupling without chance for pair production even in the presence of strong couplings. It is also shown that an accidental degeneracy appears for a particular mixing of couplings.
\end{abstract}

\section{Introduction}

The success of the Dirac equation in describing protonnucleus scattering encourages the use of others fundamental relativistic wave equations in treating other nuclear probes. In the 1930s, Duffin, Kemmer and Petiau proposed a new relativistic wave equation able to describe the dynamics of spin-zero and spin-one particles [1-4]. The Duffin-KemmerPetiau (DKP) formalism holds a large number of couplings,

\footnotetext{
a e-mail: 1rb.castro@ufma.br; luis.castro@pq.cnpq.br

be-mail: luizp@if.usp.br

ce-mail: marcelogarcia82@gmail.com

de-mail: castro@pq.cnpq.br
}

not only electromagnetic interactions, which enables one to emulate a mean field theory for describing hadron interactions. This large number of couplings makes the DKP equation a great tool for physicists as regards a phenomenological description of nuclear processes. The DKP formalism has been widely used in the description of many processes in elementary particle and nuclear physics as for instance, in the analysis of $K_{l 3}$ decays, the decay-rate ratio $\Gamma(\eta \rightarrow \gamma \gamma) / \Gamma\left(\pi^{0} \rightarrow \gamma \gamma\right)$, and level shifts and widths in pionic atoms [5-7]. Kozac and Collaborators [8] found that the DKP-based deuteron-nucleus optical potential are in close agreement with those obtained in other approachs $[9,10]$. Fischbach and co-authors definite a testable prediction of a kinematic zero at $t=\left(m_{K}+m_{\pi}\right)^{2}$ in the "effective" scalar form factor [5]. The same authors conclude that, based on others studies [11,12], the DKP equation is superior to the Klein-Gordon (KG) equation for the description of scalar particles [13]. Willey and Collaborators [14] applied the standard $S$-matrix kinematic analysis and showed that the form factors are free of kinematic singularities or constraints. Aydin and Barut [15] obtained the energy spectra and the branching ratio of the two $K_{l 3}$ modes and the $\pi_{e 3}$ decay rate with good agreement to experiment. The DKP formalism has also applications in other contexts, as such, in noncommutative phase space [16], in very special relativity (VSR) symmetries [17], in Bose-Einstein condensates $[18,19]$, in topological defects [20,21], in thermodynamics properties [22], in topological semimetals [23], in noninertial effect of rotating frames [24], among others.

Although the formalisms are equivalent in the case of minimally coupled vector interactions [25-27], the DKP formalism enjoys a richness of couplings not capable of being expressed in the KG and Proca theories [28,29]. The nonminimal vector interaction refers to a kind of charge conjugate 
invariant coupling that behaves like a vector under a Lorentz transformation. Nonminimal vector potentials, added by other kinds of Lorentz structures, have already been used in a phenomenological context for describing the scattering of mesons by nuclei [30-33], but it should be mentioned that the nonminimal vector couplings have been improperly used. Other misconception is found in Refs. [30,31], where the space component of the nonminimal vector potential is absorbed into the spinor. However, as it is shown in [34], there is no chance to discard this term. Recently, the demand for a conserved four-current has been used to point out a misleading treatment in the literature regarding analytical solutions for nonminimal vector interactions (see [35] for a comprehensive list of references). Elsewhere [36], using the proper form of the nonminimal vector coupling and considering spherically symmetric potential functions, it has been shown that the solution for the problem can be found in a clear and transparent way in terms of a Schrödinger-like equation for just one component of the DKP spinor and the remaining components are expressed in terms of that one in a simple way. Also, it has been shown that the proper boundary conditions are imposed in a simple way by observing the absence of Dirac delta potentials. On the other hand, the Coulomb problem has been intensively studied because of its intrinsic interest and also for applications in different research fields. The Coulomb problem in the context of the DKP equation has been reported in the literature for minimal vector Coulomb interaction [37-39] and for scalar Coulomb interaction [40]. To the best of our knowledge, no one has reported on the solution of scalar bosons with a full vector Coulomb interaction.

In the present work, we show the correct use of the nonminimal vector interaction in view of misconceptions propagated in the literature and we address the problem of scalar bosons embedded in a full vector Coulomb potential. We show that the Coulomb interaction (whether attractive or repulsive) leads to a Whittaker differential equation. The behavior of the scattering and bound-state solutions as well as the restrictions on the potential parameters are discussed in detail. This work treats mathematical aspects that may be important in nuclear physics but are important by themselves because they are closed analytical results. Mesons are submitted to strong interactions that are emulated on the mean field theory by a external potential. Beyond its intrinsic interest, the Coulomb interaction is a long-range like, but allows us to obtain analytical solutions that can give us a heuristic look of strong interactions in the tail, so that the new results reported in the present work are very important for a better understanding in the phenomenological description of elastic meson-nucleus scattering.

This work is organized as follows. In Sect. 2, we give a brief review on the DKP equation. We discuss the condition on the interactions which leads to a conserved current (Sect.
2.1). In Sect. 3, we concentrate our efforts on the full vector interaction. In particular, we focus on the case of scalar bosons and obtain the equation of motion, which describes the relativistic quantum dynamics (Sect. 3.1). Considering the Coulomb interaction, we find the scattering and boundstate solutions (Sects. 3.3, 3.4, respectively). Finally, in Sect. 4 we present our conclusions.

\section{Review on DKP equation}

We consider the Lagrangian density for the free DKP field (with units in which $\hbar=c=1$ )

$\mathscr{L}=\frac{i}{2}\left[\bar{\psi} \beta^{\mu}\left(\partial_{\mu} \psi\right)-\left(\partial_{\mu} \bar{\psi}\right) \beta^{\mu} \psi\right]-m \bar{\psi} \psi$

where the adjoint spinor $\bar{\psi}$ is given by $\bar{\psi}=\psi^{\dagger} \eta^{0}$ with $\eta^{0}=2 \beta^{0} \beta^{0}-1$ in such a way that $\left(\eta^{0} \beta^{\mu}\right)^{\dagger}=\eta^{0} \beta^{\mu}$ (the matrices $\beta^{\mu}$ are Hermitian with respect to $\eta^{0}$ ). The matrices $\beta^{\mu}$ satisfy the algebra

$\beta^{\mu} \beta^{v} \beta^{\lambda}+\beta^{\lambda} \beta^{v} \beta^{\mu}=g^{\mu v} \beta^{\lambda}+g^{\lambda v} \beta^{\mu}$

and the metric tensor is $g^{\mu \nu}=\operatorname{diag}(1,-1,-1,-1)$. The algebra expressed by (2) generates a set of 126 independent matrices whose irreducible representations are a trivial representation, a five-dimensional representation describing the spin-zero particles and a ten-dimensional representation associated to spin-one particles.

The equation of motion obtained from the Lagrangian (1), is given by [4]

$\left(i \beta^{\mu} \partial_{\mu}-m\right) \psi=0$

This equation is covariant under Lorentz transformation (see Appendix A)

A well-known conserved four-current is given by

$J^{\mu}=\frac{1}{2} \bar{\psi} \beta^{\mu} \psi$

where the adjoint spinor $\bar{\psi}$ is given by $\bar{\psi}=\psi^{\dagger} \eta^{0}$ with $\eta^{0}=2 \beta^{0} \beta^{0}-1$ in such a way that $\left(\eta^{0} \beta^{\mu}\right)^{\dagger}=\eta^{0} \beta^{\mu}$ (the matrices $\beta^{\mu}$ are Hermitian with respect to $\left.\eta^{0}\right)$. Despite the similarity to the Dirac equation, the DKP equation involves singular matrices, the time component of $J^{\mu}$ is not positive definite and the case of massless bosons cannot be obtained by a limiting process [41]. Nevertheless, the matrices $\beta^{\mu}$ plus the unit operator generate a ring consistent with integer-spin algebra and $J^{0}$ may be interpreted as a charge density. The factor $1 / 2$ multiplying $\bar{\psi} \beta^{\mu} \psi$, of no importance regarding the conservation law, is in order to hand over a charge density conformable to that one used in the KG theory and its 
nonrelativistic limit [34]. The normalization condition for bound-state solutions $\int \mathrm{d} \tau J^{0}= \pm 1$ can be expressed as

$$
\int \mathrm{d} \tau \bar{\psi} \beta^{0} \psi= \pm 2
$$

where the plus (minus) sign must be used for a positive (negative) charge.

\subsection{Interaction in the Duffin-Kemmer-Petiau equation}

With the introduction of interactions, the Lagrangian density for the DKP field becomes

$\mathcal{L}=\frac{i}{2}\left[\bar{\psi} \beta^{\mu}\left(\partial_{\mu} \psi\right)-\left(\partial_{\mu} \bar{\psi}\right) \beta^{\mu} \psi\right]-m \bar{\psi} \psi-\bar{\psi} U \psi$

where the more general potential matrix $U$ is written in terms of 25 (100) linearly independent matrices pertinent to five (ten)-dimensional irreducible representation associated to the scalar (vector) sector. The equation of motion obtained from the Lagrangian (6), is given by

$$
\left(i \beta^{\mu} \partial_{\mu}-m-U\right) \psi=0 .
$$

In the presence of interaction, $J^{\mu}$ satisfies the equation

$$
\partial_{\mu} J^{\mu}+\frac{i}{2} \bar{\psi}\left(U-\eta^{0} U^{\dagger} \eta^{0}\right) \psi=0 .
$$

Thus, if $U$ is Hermitian with respect to $\eta^{0}$ then four-current will be conserved. The potential matrix $U$ can be written in terms of well-defined Lorentz structures. For the spin-zero sector there are two scalar, two vector and two tensor terms [28], whereas for the spin-one sector there are two scalar, two vector, a pseudoscalar, two pseudovector and eight tensor terms [29]. The tensor terms have been avoided in applications because they furnish noncausal effects $[28,29]$. The condition (8) has been used to point out a misleading treatment in the recent literature regarding solutions for nonminimal vector interactions [35,42-44].

\section{Vector interactions in the DKP equation}

Considering only the vector terms, the DKP equation can be written as

$$
\left(i \beta^{\mu} \partial_{\mu}-m-\beta^{\mu} A_{\mu}^{(1)}-i\left[P, \beta^{\mu}\right] A_{\mu}^{(2)}\right) \psi=0
$$

where $P$ is a projection operator $\left(P^{2}=P\right.$ and $\left.P^{\dagger}=P\right)$ in such a way that $\bar{\psi}\left[P, \beta^{\mu}\right] \psi$ behaves like a vector under a Lorentz transformation as does $\bar{\psi} \beta^{\mu} \psi$. Here, $A_{\mu}^{(1)}$ and $A_{\mu}^{(2)}$ are the four-vector potential functions. Notice that the vector potential $A_{\mu}^{(1)}$ is minimally coupled but not $A_{\mu}^{(2)}$. One very important point to note is that this potential leads to a conserved four-current but the same does not happen if instead of $i\left[P, \beta^{\mu}\right]$ one uses either $P \beta^{\mu}$ or $\beta^{\mu} P$, as in [30-33]. As a matter of fact, in [30] it is mentioned that $P \beta^{\mu}$ and $\beta^{\mu} P$ produce identical results.

The invariance of the nonminimal vector potential under charge conjugation means that it does not couple to the charge of the boson. In other words, $A_{\mu}^{(2)}$ does not distinguish particles from antiparticles. Hence, whether one considers spin-zero or spin-one bosons, this sort of interaction cannot exhibit Klein's paradox [34]. On the other hand, the charge-conjugation operation changes the sign of the minimal interaction potential, i.e., changes the sign of $A_{\mu}^{(1)}$ (see Appendix B).

If the potential is time-independent one can write $\psi(\mathbf{r}, t)=\phi(\mathbf{r}) \exp (-i E t)$, where $E$ is the energy of the boson, in such a way that the time-independent DKP equation becomes

$$
\left(\beta^{0} E+i \boldsymbol{\beta} \cdot \nabla-m-\beta^{\mu} A_{\mu}^{(1)}-i\left[P, \beta^{\mu}\right] A_{\mu}^{(2)}\right) \phi=0 .
$$

In this case $J^{\mu}=\bar{\phi} \beta^{\mu} \phi / 2$ does not depend on time, so that the spinor $\phi$ describes a stationary state.

\subsection{Scalar sector}

For the case of spin-zero (scalar sector), the $\beta^{\mu}$ matrices are [44]

$\beta^{0}=\left(\begin{array}{cc}\theta & \overline{0} \\ \overline{0}^{T} & \mathbf{0}\end{array}\right), \quad \boldsymbol{\beta}=\left(\begin{array}{cc}\widetilde{0} & \boldsymbol{\rho} \\ -\boldsymbol{\rho}^{T} & \mathbf{0}\end{array}\right)$

where

$$
\begin{gathered}
\theta=\left(\begin{array}{ll}
0 & 1 \\
1 & 0
\end{array}\right), \quad \rho^{1}=\left(\begin{array}{ccc}
-1 & 0 & 0 \\
0 & 0 & 0
\end{array}\right) \\
\rho^{2}=\left(\begin{array}{ccc}
0 & -1 & 0 \\
0 & 0 & 0
\end{array}\right), \quad \rho^{3}=\left(\begin{array}{ccc}
0 & 0 & -1 \\
0 & 0 & 0
\end{array}\right)
\end{gathered}
$$

$\overline{0}, \widetilde{0}$ and $\mathbf{0}$ are $2 \times 3,2 \times 2$ and $3 \times 3$ zero matrices, respectively, while the superscript $\mathrm{T}$ designates matrix transposition. In this representation $P=\frac{1}{3}\left(\beta^{\mu} \beta_{\mu}-1\right)=\operatorname{diag}(1,0,0,0,0)$, i.e. $P$ projects out the first component of the DKP spinor. The five-component spinor can be written as $\phi^{T}=\left(\phi_{1}, \ldots, \phi_{5}\right)$ and the three-dimensional DKP equation for scalar bosons becomes

$$
\left[\left(i \nabla+\mathbf{A}^{(1)}\right)^{2}+\nabla \cdot \mathbf{A}^{(2)}+\left(\mathbf{A}^{(2)}\right)^{2}\right] \phi_{1}=k^{2} \phi_{1}
$$


$\phi_{2}=\frac{1}{m}\left(E-A_{0}^{(1)}+i A_{0}^{(2)}\right) \phi_{1}$

$\zeta=\left(\nabla-i \mathbf{A}^{(1)}-\mathbf{A}^{(2)}\right) \phi_{1}$

where

$k^{2}=\left(E-A_{0}^{(1)}\right)^{2}-m^{2}+\left(A_{0}^{(2)}\right)^{2}$

and

$\zeta=\left(\zeta_{1}, \zeta_{2}, \zeta_{3}\right)=\frac{m}{i}\left(\phi_{3}, \phi_{4}, \phi_{5}\right)$

Note that Eqs. (13), (14) and (15) clearly show that the DKP spinor has an excess of components. We can see that $\phi_{1}$ is the independent component of the DKP spinor $\phi$, which is associated to correct physical component and represents a complex scalar field [27]. The other 4 components of $\phi$ are superfluous components, which are expressed in terms of $\phi_{1}$. Meanwhile,

$$
\begin{aligned}
& J^{0}=\frac{E-A_{0}^{(1)}}{m}\left|\phi_{1}\right|^{2}, \\
& \mathbf{J}=\frac{1}{m}\left[\operatorname{Im}\left(\phi_{1}^{*} \nabla \phi_{1}\right)-\mathbf{A}^{(1)}\left|\phi_{1}\right|^{2}\right] .
\end{aligned}
$$

If we consider spherically symmetric potentials

$$
\left(A^{(\alpha)}\right)^{\mu}(\mathbf{r})=\left(A_{0}^{(\alpha)}(r), A_{r}^{(\alpha)}(r) \widehat{\mathbf{r}}\right), \quad \alpha=1,2
$$

then the DKP equation permits the factorization

$\phi_{1}(\mathbf{r})=\frac{u_{\kappa}(r) e^{i \Lambda}}{r} Y_{l m_{l}}(\theta, \varphi)$

where $A_{r}^{(1)}=\mathrm{d} \Lambda / \mathrm{d} r, Y_{l m_{l}}$ is the usual spherical harmonic, with $l=0,1,2, \ldots, m_{l}=-l,-l+1, \ldots, l$, $\int \mathrm{d} \Omega Y_{l m_{l}}^{*} Y_{l^{\prime} m_{l^{\prime}}}=\delta_{l l^{\prime}} \delta_{m_{l} m_{l^{\prime}}}$ and $\kappa$ stands for all quantum numbers which may be necessary to characterize $\phi_{1}$. For $r \neq 0$ the radial function $u$ obeys the radial equation

$\frac{\mathrm{d}^{2} u}{\mathrm{~d} r^{2}}+\left[k^{2}-2 \frac{A_{r}^{(2)}}{r}-\frac{\mathrm{d} A_{r}^{(2)}}{\mathrm{d} r}-\frac{l(l+1)}{r^{2}}-\left(A_{r}^{(2)}\right)^{2}\right] u=0$.

If the potentials $A_{0}^{(1)}, A_{0}^{(2)}$ and $A_{r}^{(2)}$ go to zero at large distances the proper solution has the asymptotic behavior $e^{i K r}$ as $r \rightarrow \infty$, with

$K=\sqrt{E^{2}-m^{2}}$.

Therefore, scattering states occur only if $K \in \mathbb{R}$, whereas bound states occur only if $K=i|K|$. Furthermore, in the case of bound-state solutions the condition $\int \mathrm{d} \tau J^{0}= \pm 1$ implies

$\frac{E}{m} \int_{0}^{\infty} \mathrm{d} r|u|^{2}-\frac{1}{m} \int_{0}^{\infty} \mathrm{d} r A_{0}^{(1)}|u|^{2}= \pm 1$.

Therefore, for motion in a central field, the solution of the three-dimensional DKP equation can be found by solving a Schrödinger-like equation. The other components are obtained from (14) and (15). Due to the spherical symmetry, Eq. (21) does not depend on $m_{l}$. Hence, for each $l$ the energy is degenerate $2 l+1$ times (essential degeneracy).

\subsection{Full vector Coulomb potential}

Let us consider the vector terms in the form

$A_{0}^{(1)}=\frac{a_{0}^{(1)}}{r}, \quad A_{0}^{(2)}=\frac{a_{0}^{(2)}}{r}, \quad A_{r}^{(2)}=\frac{a_{r}^{(2)}}{r}$.

Substituting (24) in (21), we obtain

$\frac{\mathrm{d}^{2} u}{\mathrm{~d} r^{2}}+\left[K^{2}-\frac{\alpha_{1}}{r}-\frac{\alpha_{2}+l(l+1)}{r^{2}}\right] u=0$,

with

$\alpha_{1}=2 E a_{0}^{(1)}$,

$\alpha_{2}=a_{r}^{(2)}\left(a_{r}^{(2)}+1\right)-\left(a_{0}^{(1)}\right)^{2}-\left(a_{0}^{(2)}\right)^{2}$.

Using the abbreviations

$\gamma_{l}=\sqrt{\left(l+\frac{1}{2}\right)^{2}+\alpha_{2}}$,

$\eta=\frac{\alpha_{1}}{2 K}$,

and the change $z=-2 i K r$, Eq. (25) becomes

$\frac{\mathrm{d}^{2} u}{\mathrm{~d} z^{2}}+\left(-\frac{1}{4}-\frac{i \eta}{z}+\frac{1 / 4-\gamma_{l}^{2}}{z^{2}}\right) u=0$

This second-order differential equation is the so-called Whittaker equation, which has two linearly independent solutions $M_{-i \eta, \gamma_{l}}(z)$ and $W_{-i \eta, \gamma_{l}}$ behaving like $z^{1 / 2+\gamma_{l}}$ and $z^{1 / 2-\gamma_{l}}$ close to the origin, respectively. Owing to the normalization condition expressed by Eq. (23), $u$ must behave as $r^{\epsilon}$ near the origin with $\operatorname{Re}(\epsilon)>0$ so that only the particular solution $M_{-i \eta, \gamma_{l}}(z)$ with $\gamma_{l}>0$ is allowed. The solution can be written as

$u(z)=A e^{-z / 2} z^{1 / 2+\gamma_{l}} M\left(1 / 2+\gamma_{l}+i \eta, 1+2 \gamma_{l}, z\right)$ 
where $A$ is a arbitrary constant,

$\alpha_{2}>-1 / 4$,

and $M(a, b, z)$ is the confluent hypergeometric function (Kummer's function) [45] with the asymptotic behavior for large $|z|$ and $-\frac{3 \pi}{2}<\arg z \leq-\frac{\pi}{2}[45]$

$M(a, b, z) \simeq \frac{\Gamma(b)}{\Gamma(b-a)} e^{-i \pi a} z^{-a}+\frac{\Gamma(b)}{\Gamma(a)} e^{z} z^{a-b}$.

\subsection{Scattering states}

We can show that for $|z| \gg 1$ and $K \in \mathbb{R}$ the asymptotic behavior dictated by (33) implies

$u(r) \simeq \sin \left(K r-\frac{l \pi}{2}+\delta_{l}\right)$

where the relativistic Coulomb phase shift $\delta_{l}=\delta_{l}(\eta)$ is given by

$\delta_{l}=\frac{\pi}{2}\left(l+1 / 2-\gamma_{l}\right)+\arg \Gamma\left(1 / 2+\gamma_{l}+i \eta\right)$.

For scattering states, the solution of the DKP equation (13) has the asymptotic form

$e^{-i \Lambda} \phi_{1}(\mathbf{r}) \simeq e^{i K r \cos \theta}+f(\theta, \varphi) \frac{e^{i K r}}{r}$,

where the first term represents a plane wave moving along the direction $\theta=0$ toward the scatterer, and the second term represents a radially outgoing wave. For spherically symmetric scatterers, both terms exhibit cylindrical symmetry about the direction of incidence in such a way that $\phi_{1}$ and $f$ are independent of $\varphi$. The connection between the forms (20) and (36) allows us to write the scattering amplitude as a partial wave series

$f(\theta)=\sum_{l=0}^{\infty}(2 l+1) f_{l} P_{l}(\cos \theta)$,

where $P_{l}$ is the Legendre polynomial of order $l$ and the partial scattering amplitude is $f_{l}=\left(e^{2 i \delta_{l}}-1\right) /(2 i K)$. With the phase shift (35), up to a logarithmic phase inherent to the Coulomb field, we find

$2 i K f_{l}=-1+e^{i \pi\left(l+1 / 2-\gamma_{l}\right)} \frac{\Gamma\left(1 / 2+\gamma_{l}+i \eta\right)}{\Gamma\left(1 / 2+\gamma_{l}-i \eta\right)}$.

The series (37) can be summed only when $\gamma_{l}=l+1 / 2$, i.e. when

$\alpha_{2}=0$.
The closed form being [46]

$f(\theta)=-\eta \frac{\Gamma(1+i \eta)}{\Gamma(1-i \eta)} \frac{e^{-i \eta \ln ^{2} \sin ^{2} \theta / 2}}{2 K \sin ^{2} \theta / 2}, \quad \theta \neq 0$,

which gives the well-known Rutherford scattering formula for the differential cross section in classical and nonrelativistic quantum mechanics. This happens because the condition (39) implies that the scalar boson is effectively under the influence of a Coulomb potential (without inversely quadratic terms).

\subsection{Bound states}

If $K=i|K|$, the partial scattering amplitude becomes infinite when $1 / 2+\gamma_{l}+i \eta=-n$, where $n=0,1,2, \ldots$, due to the poles of the gamma function in the numerator of (38), and (33) implies that $u$ tends to $r^{1 / 2+\gamma_{l}+n} e^{-|K| r}$ for large $r$. Therefore, bound-state solutions are possible only for $\alpha_{1}<0$, i.e., when $E \gtrless 0$ and $a_{0}^{(1)} \lessgtr 0$ and the spectrum is expressed as

$$
E=-\frac{\operatorname{sgn}\left(a_{0}^{(1)}\right) m}{\sqrt{1+\frac{\left(a_{0}^{(1)}\right)^{2}}{\left(n+\gamma_{l}+1 / 2\right)^{2}}}} .
$$

Because $M(-n, b, z)$ is proportional to the generalized Laguerre polynomial $L_{n}^{(b-1)}(z)$ [45] and using a pair of integral formulas involving $L_{n}^{(b-1)}(z)$ [47] one can finally write $\phi_{1}$ as

$$
\begin{aligned}
\phi_{1}(\mathbf{r})= & N e^{i \Lambda} r^{\gamma_{l}-1 / 2} e^{-\frac{\left|\alpha_{1}\right| r}{2\left(n+\gamma_{l}+1 / 2\right)}} \\
& \times L_{n}^{\left(2 \gamma_{l}\right)}\left(\frac{\left|\alpha_{1}\right| r}{n+\gamma_{l}+1 / 2}\right) Y_{l m_{l}}(\theta, \varphi)
\end{aligned}
$$

with

$N=\sqrt{\frac{|E|}{m} \frac{2^{2 \gamma_{l}+1}|K|^{2 \gamma_{l}+2}}{\left(n+\gamma_{l}+1 / 2\right)} \frac{n !}{\Gamma\left(n+2 \gamma_{l}+1\right)}}$

where the radial quantum number $n$ is related to the number of zeros of $\phi_{1}$. In addition to the essential degeneracy mentioned before, accidental degeneracy exists when $\alpha_{2}=0$. In this case $E$ depends on $n$ and $l$ through the combination $n+l$ in such a way that each energy is $(n+l+1)^{2}$-fold degenerate.

\section{Conclusions}

In this work, we have addressed the relativistic quantum dynamics of scalar bosons embedded in a full vector Coulomb interaction in the context of the DKP formalism. 
We showed that using the proper form of the full vector interaction and considering a Coulomb potential (whether attractive or repulsive) the scattering and bound-state solutions can be obtained by solving a Whittaker differential equation.

For scattering solutions, we calculated the relativistic Coulomb phase shift and expressed the scattering amplitude as a partial wave sum for the general case of vector interactions. The space component of the minimal coupling contributes only with an $l$-independent phase factor in the partial wave. The scattering amplitude can be summed only when $\gamma_{l}=l+1 / 2\left(\alpha_{2}=0\right)$. In this particular case, the closed form leads to the Rutherford scattering formula for the differential cross section. Incidentally, the Rutherford scattering formula requires the presence of the space component of the nonminimal coupling and it is valid even if the interactions are strong. Furthermore, the Rutherford cross section vanishes in the absence of the time component of the minimal coupling making the interaction transparent. Of course, our results are valid in the approximation $\gamma_{l} \simeq l+1 / 2\left(\alpha_{2} \simeq 0\right)$.

On the other hand, the existence of bound-state solutions requires the presence of the time component of the minimal coupling $\left(a_{0}^{(1)} \neq 0\right)$, binding particles (antiparticles) with positive (negative) energies in the case of an attractive (a repulsive) potential and so the spontaneous pair production is not a possibility. The usual accidental degeneracy in the bound-state spectrum only exists when the potential parameters obey the very same restriction that leads the closed-form amplitude scattering $\left(\alpha_{2}=0\right.$, or $\alpha_{2} \simeq 0$ in a approximation scheme).

We showed that the many ways that a Coulomb potential can couple to bosons in the DKP formalism make a difference in hadronic process. We believe that the results reported in the present work are an important contribution for a better understanding of those phenomenological descriptions by the DKP formalism. A more detailed study of the interaction between mesons and nucleus can be accomplished by adding a short-range phenomenological potential apart from long-range. A refined calculation of this process should use computational methods to obtain phase-shifts which can be compared with our results obtained in a closed form. Finally, it is worthwhile to mention that the DKP formalism holds a large number of couplings, not only electromagnetic interactions, which enables one to emulate a mean field theory for describing the hadron interactions. This large number of couplings makes the DKP formalism a great tool for physicists as regards a phenomenological description of nuclear processes. Our results can be seen as a first step for future applications in nuclear processes, this is currently under study and will be reported elsewhere.

Acknowledgements The authors would like to thank the referees for useful comments and suggestions. This work was supported in part by means of funds provided by CNPq, Brazil, Grants No. 455719/2014-4
(Universal), No. 304105/2014-7 (PQ) and No. 304743/2015-1 (PQ) and CAPES, Brazil.

Open Access This article is distributed under the terms of the Creative Commons Attribution 4.0 International License (http://creativecomm ons.org/licenses/by/4.0/), which permits unrestricted use, distribution, and reproduction in any medium, provided you give appropriate credit to the original author(s) and the source, provide a link to the Creative Commons license, and indicate if changes were made.

Funded by SCOAP ${ }^{3}$.

\section{Appendix A: Lorentz covariance of the DKP equation}

Under a Lorentz transformation $x^{\prime \mu}=\Lambda^{\mu}{ }_{\nu} x^{\nu}$ we have

$$
\begin{aligned}
\psi^{\prime} & =U(\Lambda) \psi, \\
U^{-1} \beta^{\mu} U & =\Lambda^{\mu}{ }_{\nu} \beta^{\nu} .
\end{aligned}
$$

For the case of an infinitesimal transformation, we have $\Lambda_{\nu}^{\mu}=\delta_{\nu}^{\mu}+\Delta \omega_{v}^{\mu}\left(\Delta \omega_{\mu v}=-\Delta \omega_{v \mu}\right)$ with

$U=1+\frac{1}{2} \Delta_{\mu \nu} S^{\mu \nu}$

where $S^{\mu \nu}=\left[\beta^{\mu}, \beta^{\nu}\right]$ and for a finite Lorentz transformation we obtain

$U=\exp \left(\frac{1}{2} \Delta \omega_{\mu \nu} S^{\mu \nu}\right)$.

\section{Appendix A.1: Scalar sector}

To select the physical component of the DKP field for the scalar sector (spin-0 sector), we define the operators [48]

$P \equiv-\left(\beta^{0}\right)^{2}\left(\beta^{1}\right)^{2}\left(\beta^{2}\right)^{2}\left(\beta^{3}\right)^{2}, \quad P^{\mu} \equiv P \beta^{\mu}$

which satisfy

$$
\begin{aligned}
P^{2} & =P, \\
P^{\mu} \beta^{\nu} & =P g^{\mu \nu}, \\
P S^{\mu \nu} & =S^{\mu \nu} P=0, \\
P^{\mu} S^{\nu \lambda} & =\eta^{\mu \nu} P^{\lambda}-\eta^{\mu \lambda} P^{\nu} .
\end{aligned}
$$

As shown in [48]

$$
\begin{aligned}
(P \psi)^{\prime} & =P \psi, \\
\left(P^{\mu} \psi\right)^{\prime} & =\Lambda_{\lambda}^{\mu} P^{\lambda} \psi,
\end{aligned}
$$

so that $P \psi$ and $P^{\mu} \psi$ transform as a (pseudo) scalar and a (pseudo) vector under an infinitesimal Lorentz transformation, respectively. Applying $P$ and $P^{\mu}$ to the DKP equation (3) and combining the results, we get

$$
\left(\partial_{\mu} \partial^{\mu}+m^{2}\right)(P \psi)=0
$$


This result tell us that all elements of the column matrix $P \psi$ obey the KG equation. Then, acting with $P$ upon the spinor DKP $\psi$ selects the scalar sector of DKP theory, making explicitly clear that it describes a spin- 0 particle. Following this innovative view of the DKP spinor, Ref. [26] shows that the redundant components of $\psi$ are projected out, $\psi$ and $P \psi$ are both compatible with gauge invariance.

\section{Appendix A.2: Vector sector}

Now we discuss the vector sector (spin-1 sector) of the DKP theory. Similar to the scalar sector, we can select the physical components of the DKP field for the spin-1 sector, so we define the operators

$R^{\mu} \equiv\left(\beta^{1}\right)^{2}\left(\beta^{2}\right)^{2}\left(\beta^{3}\right)^{2}\left(\beta^{\mu} \beta^{0}-g^{\mu 0}\right), \quad R^{\mu \nu} \equiv R^{\mu} \beta^{\nu}$,

which satisfy

$$
\begin{aligned}
R^{\mu \nu} & =-R^{\nu \mu}, \\
R^{\mu \nu} \beta^{\alpha} & =R^{\mu} g^{\nu \alpha}-R^{\nu} g^{\mu \alpha}, \\
R^{\mu} S^{\nu \lambda} & =g^{\mu \nu} R^{\lambda}-g^{\mu \lambda} R^{\nu}, \\
R^{\mu \nu} S^{\alpha \beta} & =g^{\nu \alpha} R^{\mu \beta}-g^{\mu \alpha} R^{\nu \beta}-g^{\nu \beta} R^{\mu \alpha}+g^{\mu \beta} R^{\nu \alpha} .
\end{aligned}
$$

As shown in [48]

$$
\begin{aligned}
\left(R^{\mu} \psi\right)^{\prime} & =\Lambda_{\lambda}^{\mu}{ }_{\lambda} R^{\lambda} \psi, \\
\left(R^{\mu \nu} \psi\right)^{\prime} & =\Lambda_{\lambda}^{\mu}{ }_{\lambda}{ }^{\nu}{ }_{\beta} R^{\alpha \beta} \psi,
\end{aligned}
$$

so that $R^{\mu} \psi$ and $R^{\mu \nu} \psi$ transform as (pseudo) vector and (pseudo) tensor quantities under an infinitesimal Lorentz transformation, respectively. Again, applying $R^{\mu}$ and $R^{\mu v}$ to the DKP equation (3) and combining the results, we obtain

$\partial_{\nu} U^{v \mu}+m^{2} R^{\mu} \psi=0$

$U^{\mu \nu}=\partial^{\mu} R^{v} \psi-\partial^{\nu} R^{\mu} \psi$.

These results tell us that all elements of the column matrix $R^{\mu} \psi$ obey the Proca equation. So, similar to the scalar sector, this procedure selects the vector sector of the DKP theory, making explicitly clear that it describes a spin-1 particle.

\section{Appendix B: Symmetries of the DKP equation}

\section{Appendix B.1: Parity}

The DKP equation is invariant under the parity operation, i.e. when $\mathbf{r} \rightarrow-\mathbf{r}$, if $A_{i}^{(1)}$ and $A_{i}^{(2)}$ change sign, whereas $A_{0}^{(1)}$ and $A_{0}^{(2)}$ remain the same. This happens due to the parity operator is

$\mathcal{P}=\exp \left(i \delta_{P}\right) P_{0} \eta^{0}$,

where $\delta_{P}$ is a constant phase and $P_{0}$ changes $\mathbf{r}$ into $-\mathbf{r}$. Because this unitary operator anticommutes with $\beta^{i}$ and $\left[P, \beta^{i}\right]$, they change sign under a parity transformation, whereas $\beta^{0}$ and $\left[P, \beta^{0}\right]$, which commute with $\eta^{0}$, remain the same. Since $\delta_{P}=0$ or $\delta_{P}=\pi$, the spinor components have definite parities.

\section{Appendix B.2: Charge conjugation}

The charge-conjugation operation changes the sign of the minimal interaction potential, i.e. changes the sign of $A_{\mu}^{(1)}$. This can be accomplished by the transformation $\psi \rightarrow \psi_{C}=$ $\mathcal{C} \psi=C K \psi$, where $K$ denotes the complex conjugation and $C$ is a unitary matrix such that $C \beta^{\mu}=-\beta^{\mu} C$. The matrix that satisfies this relation is

$C=\exp \left(i \delta_{C}\right) \eta^{0} \eta^{1}$

The phase factor $\exp \left(i \delta_{C}\right)$ is equal to \pm 1 ; therefore $E \rightarrow$ $-E$. Note also that $J^{\mu} \rightarrow-J^{\mu}$, as should be expected for a charge current. Meanwhile $C$ anticommutes with $\left[P, \beta^{\mu}\right]$ and the charge-conjugation operation entails no change on $A_{\mu}^{(2)}$.

\section{Appendix B.3: Time reversal}

The DKP equation is invariant under the time-reversal transformation, i.e. when $t \rightarrow-t$, if $A_{i}^{(1)}$ and $A_{0}^{(2)}$ change sign, whereas $A_{0}^{(1)}$ and $A_{i}^{(2)}$ remain the same. This is because the time-reversal operator is

$\mathscr{T}=\exp \left(i \delta_{T}\right) T_{0} \eta^{0}$

where $\delta_{T}$ is a constant phase and $T_{0}$ denotes the complex conjugation and changes $t$ into $-t$. Because this unitary operator anticommutes with $\beta^{i}$ and $\left[P, \beta^{i}\right]$, they change sign under a time-reversal transformation, whereas $\beta^{0}$ and $\left[P, \beta^{0}\right]$ remain the same.

\section{Appendix B.4: $\mathcal{P C} \mathcal{T}$}

The DKP equation is invariant under the $\mathcal{P C} \mathcal{T}$ transformation, if both sorts of vector potential, change sign. Our results can be summarized in Table 1. 
Table 1 Summary of the results for the behavior of the minimal and nonminimal vector interactions under $\mathcal{P}, \mathcal{C}, \mathcal{T}$ and $\mathcal{P C} \mathcal{T}$. Here "+" and "-" mean "no change sign" and "change sign", respectively

\begin{tabular}{lllll}
\hline Vector interaction & $\mathcal{P}$ & $\mathcal{C}$ & $\mathcal{T}$ & $\mathcal{P C T}$ \\
\hline$A_{0}^{(1)}$ & + & - & + & - \\
$A_{i}^{(1)}$ & - & - & - & - \\
$A_{0}^{(2)}$ & + & + & - & - \\
$A_{i}^{(2)}$ & - & + & + & - \\
\hline
\end{tabular}

\section{References}

1. G. Petiau, Published in Acad. Roy. de Belg., Classe Sci., Mem in 8o 16, 2 (1936)

2. N. Kemmer, Proc. R. Soc. Lond. A 166, 127 (1938). doi:10.1098/ rspa.1938.0084

3. R.J. Duffin, Phys. Rev. 54, 1114 (1938). doi:10.1103/PhysRev.54. 1114

4. N. Kemmer, Proc. R. Soc. Lond. A 173, 91 (1939). doi:10.1098/ rspa.1939.0131

5. E. Fischbach, F. Iachello, A. Lande, M.M. Nieto, C.K. Scott, Phys. Rev. Lett. 26, 1200 (1971). doi:10.1103/PhysRevLett.26.1200

6. E. Fischbach, M.M. Nieto, C.K. Scott, Prog. Theor. Phys. 51, 1585 (1974). doi:10.1143/PTP.51.1585

7. E. Friedman, G. Kälbermann, C.J. Batty, Phys. Rev. C 34, 2244 (1986). doi:10.1103/PhysRevC.34.2244

8. R.E. Kozack, B.C. Clark, S. Hama, V.K. Mishra, G. Kälbermann, R.L. Mercer, L. Ray, Phys. Rev. C 37, 2898 (1988). doi:10.1103/ PhysRevC.37.2898

9. I.S. on Polarization Phenomena in Nuclear Physics, M. Kondo, N.B. Gakkai., Proceedings of the Sixth International Symposium on Polarization Phenomena in Nuclear Physics, Osaka, 26-30 August, 1985/edited by M. Kondo ... [et al.]. Physical Society of Japan Tokyo, 1986

10. S. Watanabe, Nucl. Phys. 8, 484 (1958). doi:10.1016/ 0029-5582(58)90180-9

11. E. Fischbach, M.M. Nieto, C.K. Scott, Phys. Rev. D 6, 726 (1972). doi:10.1103/PhysRevD.6.726

12. E. Fischbach, M.M. Nieto, C.K. Scott, Phys. Rev. D 7, 207 (1973). doi:10.1103/PhysRevD.7.207

13. E. Fischbach, M.M. Nieto, C.K. Scott, Phys. Rev. D 7, 3544 (1973). doi:10.1103/PhysRevD.7.3544

14. R.S. Willey, P. Winternitz, T. Yao, Phys. Rev. D 7, 3540 (1973). doi:10.1103/PhysRevD.7.3540

15. Z.Z. Aydin, A.O. Barut, Phys. Rev. D 7, 3522 (1973). doi:10.1103/ PhysRevD.7.3522

16. H. Hassanabadi, Z. Molaee, S. Zarrinkamar, Eur. Phys. J. C 72, 2217 (2012). doi:10.1140/epjc/s10052-012-2217-5

17. R.M.T. Cavalcanti, J.M. Hoff da Silva, R.A. da Rocha, Eur. Phys. J. Plus 129(11), 246 (2014). doi:10.1140/epjp/i2014-14246-4

18. R. Casana, V.Y. Fainberg, B.M. Pimentel, J.S. Valverde, Phys. Lett. A 316(1), 33 (2003). doi:10.1016/S0375-9601(03)01018-1

19. L.M. Abreu, A.L. Gadelha, B.M. Pimentel, E.S. Santos, Phys A Stat Mech Appl 419, 612 (2015). doi:10.1016/j.physa.2014.10.049
20. L.B. Castro, Eur. Phys. J. C 75(6), 287 (2015). doi:10.1140/epjc/ s10052-015-3507-5

21. N. Messai, A. Boumali, Eur. Phys. J. Plus 130(7), 140 (2015). doi:10.1140/epjp/i2015-15140-3

22. Z. Wang, Z.W. Long, C.Y. Long, W. Zhang, AdHEP 2015, Article ID 901675 (2015). doi:10.1155/2015/901675

23. G. Palumbo, K. Meichanetzidis, Phys. Rev. B 92, 235106 (2015). doi:10.1103/PhysRevB.92.235106

24. L.B. Castro, Eur. Phys. J. C 76(2), 61 (2016). doi:10.1140/epjc/ s10052-016-3904-4

25. M. Nowakowski, Phys. Lett. A 244, 329 (1998). doi:10.1016/ S0375-9601(98)00365-X

26. J.T. Lunardi, B.M. Pimentel, R.G. Teixeira, J.S. Valverde, Phys. Lett. A 268, 165 (2000). doi:10.1016/S0375-9601(00)00163-8

27. L.B. Castro, A.S. de Castro, Phys. Rev. A 90, 022101 (2014). doi:10.1103/PhysRevA.90.022101

28. R.F. Guertin, T.L. Wilson, Phys. Rev. D 15, 1518 (1977). doi:10. 1103/PhysRevD.15.1518

29. B. Vijayalakshmi, M. Seetharaman, P.M. Mathews, J. Phys. A Math. Gen. 12, 665 (1979). doi:10.1088/0305-4470/12/5/015

30. B.C. Clark, S. Hama, G.R. Kälbermann, R.L. Mercer, L. Ray, Phys Rev. Lett. 55, 592 (1985). doi:10.1103/PhysRevLett.55.592

31. G. Kälbermann, Phys. Rev. C 34, 2240 (1986). doi:10.1103/ PhysRevC.34.2240

32. R.C. Barrett, Y. Nedjadi, Nucl. Phys. A 585, 311 (1995). doi:10. 1016/0375-9474(94)00582-8

33. B. Clark, R. Furnstahl, L. Kerr, J. Rusnak, S. Hama, Phys. Lett. B 427, 231 (1998). doi:10.1016/S0370-2693(98)00352-9

34. T.R. Cardoso, L.B. Castro, A.S. de Castro, J. Phys. A Math. Theor. 43, 055306 (2010). doi:10.1088/1751-8113/43/5/055306

35. L.B. Castro, L.P. de Oliveira, AdHEP 2014, Article ID 784072 (2014). doi:10.1155/2014/784072

36. L.B. Castro, A.S. de Castro, Phys. Lett. A 375, 2596 (2011). doi:10. 1016/j.physleta.2011.05.067

37. Y. Nedjadi, R.C. Barrett, J. Phys. G Nucl. Part. Phys. 19, 87 (1993). doi:10.1088/0954-3899/19/1/006

38. Y. Nedjadi, R.C. Barrett, J. Math. Phys. 35, 4517 (1994). doi:10. 1063/1.530801

39. D. Owen, R. Barrett, Phys. Lett. B 574, 197 (2003). doi:10.1016/ j.physletb.2003.09.028

40. H. Hassanabadi, B.H. Yazarloo, S. Zarrinkamar, A.A. Rajabi, Phys. Rev. C 84, 064003 (2011). doi:10.1103/PhysRevC.84.064003

41. R.A. Krajcik, M.M. Nieto, Phys. Rev. D 10, 4049 (1974). doi:10. 1103/PhysRevD.10.4049

42. T.R. Cardoso, L.B. Castro, A.S. de Castro, Can. J. Phys. 87, 857 (2009). doi:10.1139/P09-054

43. T.R. Cardoso, L.B. Castro, A.S. de Castro, Can. J. Phys. 87, 1185 (2009). doi:10.1139/P09-082

44. T.R. Cardoso, L.B. Castro, A.S. de Castro, J. Phys. A Math. Theor 45, 075302 (2012). doi:10.1088/1751-8113/45/7/075302

45. M. Abramowitz, I.A. Stegun, Handbook of Mathematical Functions (Dover, Toronto, 1965)

46. M.G. Garcia, A.S. de Castro, Phys. Rev. C 91, 034903 (2015). doi:10.1103/PhysRevC.91.034903

47. G.B. Arfken, H.J. Weber, Mathematical Methods for Physicists, 5th edn. (Harcourt/Academic Press, San Diego, 1996)

48. H. Umezawa, Quantum Field Theory (North-Holland, Amsterdam, 1956) 